\title{
ERRATUM
}

Evgeni Trofimov • Anna Vainchtein

\section{Erratum to: Shocks versus kinks in a discrete model of displacive phase transitions}

Published online: 3 November 2012

(C) Springer-Verlag Berlin Heidelberg 2012

Erratum to: Continuum Mech. Thermodyn. (2010) 22:317-344

DOI 10.1007/s00161-010-0148-7

The expressions for $f_{\alpha, C}^{ \pm}(k, V)$ on p. 327 that are used to derive the asymptotics (39) and (41) are not written correctly in the published article. The correct expressions are

$$
f_{\alpha, C}^{ \pm}(k, V)=i^{1+\left|\mathcal{N}_{\alpha}^{-}\right|-\left|\mathcal{N}_{\alpha}^{+}\right|} \frac{V \Pi_{\alpha}^{+} \Pi_{\alpha}^{-}}{\sqrt{c_{\alpha}^{2}-V^{2}}}(0 \mp i k)^{-\left|\mathcal{N}_{\alpha}^{-}\right|}(0 \mp i k)^{-\left|\mathcal{N}_{\alpha}^{+}\right|} F_{\alpha}^{ \pm}(k, V),
$$

with the asymptotic behavior

$$
f_{\alpha, C}^{ \pm}(k, V) \approx i^{1+\left|\mathcal{N}_{\alpha}^{-}\right|-\left|\mathcal{N}_{\alpha}^{+}\right|} \frac{V \Pi_{\alpha}^{+} \Pi_{\alpha}^{-}}{\sqrt{c_{\alpha}^{2}-V^{2}}}(0 \mp i k)^{-\left|\mathcal{N}_{\alpha}^{-}\right|}(0 \mp i k)^{-\left|\mathcal{N}_{\alpha}^{+}\right|}, \quad k \rightarrow \pm i \infty .
$$

Elsewhere in the published article, there are some typographical errors. There is $w$ missing in the left-hand side $w(\xi)$ of (48) on p. 328, and in (49) $i \neq j$ should be replaced by $k_{\gamma, j} \neq k$ for $P_{+}(k, V)$ and $k_{1, j} \neq k$ for $P_{-}(k, V)$, i.e.

$$
P_{+}(k, V)=\frac{\prod_{k_{1, j} \in \mathcal{M}_{1}^{+}(V)}\left(1-\frac{k}{k_{1, j}}\right)}{\prod_{\substack{k_{\gamma, j} \in \mathcal{M}_{\gamma}^{+}(V), k_{\gamma, j} \neq k}}\left(1-\frac{k}{k_{\gamma, j}}\right)}, \quad P_{-}(k, V)=\frac{\prod_{k_{\gamma, j} \in \mathcal{M}_{\gamma}^{-}(V)}\left(1-\frac{k}{k_{\gamma, j}}\right)}{\prod_{\substack{k_{1, j} \in \mathcal{M}_{1}^{-}(V), k_{1, j} \neq k}}\left(1-\frac{k}{k_{1, j}}\right)} .
$$

The online version of the original article can be found under doi:10.1007/s00161-010-0148-7.

E. Trofimov · A. Vainchtein $(\varangle)$

Department of Mathematics, University of Pittsburgh, Pittsburgh, PA 15260, USA

E-mail: aav4@pitt.edu

E. Trofimov

E-mail: evt3@pitt.edu 
On p. 331, in the second line of (62) (for $\xi>0$ ) $e^{-i k_{\gamma, i} \xi}$ should be replaced by $e^{-i k_{1, i} \xi}$. On p. 332, in the second expression for $I_{+}, 1 / \mathcal{R}(V)$ should be replaced by $\sqrt{L_{0}(V)}$. In the first expression of the equation immediately after it, $\gamma$ should be replaced by 1 , i.e.

$$
\sum_{k_{1, i} \in \mathcal{M}_{1}^{-}(V)} k_{1, i} P_{-}\left(k_{1, i}, V\right)=-\mathcal{R}(V) \sqrt{L_{0}(V)} .
$$

We also note that the first paragraph of 7.3 on p. 335 is not clearly written. As can be seen from the solution (48), only the roots from $\mathcal{N}_{\gamma}^{+}$and $\mathcal{N}_{1}^{-}$are the wave numbers of the non-decaying lattice waves propagating behind and ahead, respectively, of the moving front. The other roots contribute to the structure of these waves. 